\title{
Risk factors of atherosclerosis in elderly senile obstructive sleep apnea hypopnea syndrome patients with hypertension and type 2 diabetes
}

\author{
Gaisheng Tian, Lingling Feng* \\ Department of Geriatrics, The Third Affiliated Hospital of Inner Mongolia Medical University, Baotou, Inner Mongolia, China
}

Received: September 12, 2017

Accepted: October 25, 2017

Online Published: December 10, 2017

DOI: $10.14725 / d c c . v 4 n 4 p 1$

URL: http://dx.doi.org/10.14725/dcc.v4n4p1

\begin{abstract}
Objective: To explore the related factors of atherosclerosis in senile obstructive sleep apnea hypopnea syndrome (OSAHS) patients.

Methods: 129 outpatients and inpatients with senile hypertension and type 2 diabetes were chosen from Department of Geriatrics during May of 2012 to January of 2014. The sleep breath monitoring was performed to each patient in the early stage. According to the presence or absence of OSAHS, they were divided into OSAHS $(n=60)$ and non-OSAHS $(n=69)$ groups. And 30 healthy elderly subjects recruited in Physical Examination Department of our hospital were included in the control group. The parameters of age, gender, height, body mass index, hypersensitive C-reactive protein (Hs-CRP), homocysteine (Hcy), triglyceride (TG), total cholesterol (TC), high density lipoprotein-cholesterol (HDL-C), low density lipoprotein-cholesterol (LDL-C) and carotid intima-media thickness (CIMT) were recorded. The comparison of Hs-CRP, Hcy, TG, TC, HDL-C, LDL-C and CIMT between OSAHS and non-OSAHS groups was made; and the comparison with the normal control group was made to analyze the correlations of CIMT to the above-mentioned indexes.

Results: The levels of Hs-CRP [(3.40 \pm 0.91$) \mathrm{mg} / \mathrm{L},(2.57 \pm 1.01) \mathrm{mg} / \mathrm{L}]$, Hcy $[(16.50 \pm 2.89) \mu \mathrm{mol} / \mathrm{L},(11.20 \pm 2.32) \mu \mathrm{mol} / \mathrm{L}]$, CIMT $[(1.06 \pm 0.14) \mathrm{mm},(0.93 \pm 0.13) \mathrm{mm}], \mathrm{TG}[(2.52 \pm 1.40) \mathrm{mmol} / \mathrm{L},(2.51 \pm 2.02) \mathrm{mmol} / \mathrm{L}], \mathrm{TC}[(5.74 \pm 1.16) \mathrm{mmol} / \mathrm{L}$, $(5.49 \pm 1.09) \mathrm{mmol} / \mathrm{L}]$ and LDL-C $[(3.15 \pm 0.47) \mathrm{mmol} / \mathrm{L},(3.05 \pm 0.70) \mathrm{mmol} / \mathrm{L}]$ in both OSAHS and non-OSAHS groups were higher than those in normal control group [Hs-CRP: $(2.06 \pm 1.22) \mathrm{mg} / \mathrm{L}$, Hcy: $(6.04 \pm 1.85) \mu \mathrm{mol} / \mathrm{L}$, CIMT: $(0.65 \pm 0.07) \mathrm{mm}$, TG: $(0.99 \pm 0.24) \mathrm{mmol} / \mathrm{L}$, TC: $(3.63 \pm 0.71) \mathrm{mmol} / \mathrm{L}$ and LDL-C: $(2.47 \pm 0.27) \mathrm{mmol} / \mathrm{L}]$; the levels of Hs-CRP, Hcy, CIMT and plaque score in OSAHS group were higher than those in non-OSAHS group [plague score: $(1.92 \pm 1.03)$ vs. $(1.35 \pm 0.97)$ points], the difference was of statistical significance (OSAHS group vs. control group, $t=5.88,18.15,15.62,5.91,9.17,7.47$; non-OSAHS group vs. control group, $t=2.20,10.73,11.51,4.09,8.56,4.45$; OSAHS group vs. non-OSAHS group, $t=4.84$, 11.72, 5.54, 3.91, all $p<.05$ ). Related analyses showed that CIMT and plaque score in OSAHS group were correlated to age, BMI, TG, TC, LDL-C, Hs-CRP and Hcy respectively.

Conclusions: OSAHS may elevate the level of inflammatory cytokines and cause atherosclerosis. And the changes of CIMT, Hs-CRP and Hcy are in parallel. Hcy also has an identically important clinical significance in the progress of atherosclerosis.
\end{abstract}

Key Words: Sleep apnea, Obstructive, C-reactive protein, Homocysteine

\footnotetext{
*Correspondence: Lingling Feng; E-mail: bgyytgs@ 163.com; Address: Department of Geriatrics, The Third Affiliated Hospital of Inner Mongolia Medical University, Baotou, Inner Mongolia, China.
} 
Foreign literatures have reported that the incidence of obstructive sleep apnea hypopnea syndrome (OSAHS) in the elderly ranges from $20 \%$ to $40 \% .^{[1]}$ The carotid intimamedia thickness (CIMT) can be used as a window to evaluate atherosclerosis (AS), ${ }^{[2]}$ which is easily detected by ultrasonography that has been widely used to evaluate the progress and severity of AS. Recent literatures show that OSAHS shares some common pathophysiologic mechanisms with AS. ${ }^{[3]}$ AS is a chronic inflammatory disease caused by vascular endothelial damage, and inflammation is involved in the occurrence and development of AS as well as the whole course of its complications. ${ }^{[4-6]}$ Hypersensitive C-reactive protein (Hs-CRP) is an inflammatory marker which is the most widely studied in the current risk prediction of cardiovascular events. Homocysteine (Hcy) is thought to be a type of inflammatory stimuli. When the level of Hcy is increased, Hcy will activate a variety of inflammatory cytokines in the body, where it will directly or indirectly involve itself in the inflammatory process and lead to AS eventually. This study explored the changes of Hs-CRP and Hcy and the correlation to AS under the condition of hypoxia.

\section{Objects and methods}

\section{$1.1 \quad$ Research objects}

129 outpatients and inpatients with senile hypertension and type 2 diabetes were chosen from Department of Geriatrics during May of 2012 to January of 2014. The sleep breath monitoring was performed to each patient in the early stage. According to the presence or absence of OSAHS, they were divided into OSAHS $(\mathrm{n}=60)$ and non-OSAHS $(\mathrm{n}=69)$ groups. Among patients in OSAHS group, the numbers of patients only with hypertension, patients only with type 2 diabetes, patients with hypertension and type 2 diabetes as well as patients with hypertension, type 2 diabetes and coronary disease were 14, 13, 30 and 3; among patients in non-OSAHS group, the numbers of patients only with hypertension, patients only with type 2 diabetes, patients with hypertension and type 2 diabetes as well as patients with hypertension, type 2 diabetes and coronary disease were 16 , 18, 31 and 4. During the same period, healthy elders (30 cases) selected from our Physical Examination Department were included in normal control group. All of them, over 60 years old, had a history of snoring, and did not receive the treatment of diuretics or heparin and oral vitamin B and folic acid. Exclusion criteria were as follows: patients with chronic obstructive pulmonary disease, a variety of recent acute and chronic infections, autoimmune diseases and tumors. All patients were well informed and signed informed consent forms.

\subsection{Research methods}

Basic information, including age, gender, snoring history, height, body mass index and so on, was recorded. Polysomnography [CB Type (portable) polysomnography system made by Curative Medical Science and Technology Co., Ltd.] was conducted between 10 p.m. and 7 a.m. the next day. No alcohol, coffee, tranquilizers and hypnotics were taken on the day of detection. SW-SM2000CB polysomnography diagnostic and analytical system was applied to acquisition, display, storage, analysis of multichannel physiological data and diagnosis of OSAHS. TG, TC, HDL-C, LDL-C, Hs-CRP and Hcy were measured subsequently. 4 indexes of TG, TC, HDL-C and LDL$\mathrm{C}$ were measured by use of immune colorimetry, Hs-CRP was measured with immune transmission turbidimetry, and Hcy was measured by means of immunoluminometric assay. Bilateral common carotid arteries, the bifurcation of common carotid artery and the initial segment of external carotid artery were scanned and detected by use of Philips iE33 Color Doppler Ultrasonic Diagnosis Apparatus with the probe frequency of $10 \mathrm{MHz}$. CIMT was measured at a distance of $1.0 \mathrm{~cm}$ proximal to the bifurcation of common carotid artery, with the presence or absence of plaques confirmed at the same time. The average value of CIMT (calculated by CIMT values of the left and the right common carotid arteries, internal carotid arteries and external carotid arteries) was selected as the experimental CIMT. The carotid plaques were evaluated by use of semiquantitative scoring method, with the score ranging from 0 to 4: 0 stands for no plaque; 1 for 1 plaque with the thickness less than $2 \mathrm{~mm}$; 2 for 2 plaques with the thickness no more than $2 \mathrm{~mm}$, or 1 plaque with the thickness more than $2 \mathrm{~mm} ; 3$ represents 2 plaques, with the thickness of at least 1 plaque more than $2 \mathrm{~mm}$; 4 represents at least 2 plaques, whose thickness are all more than $2 \mathrm{~mm} .{ }^{[7]}$ OSAHS diagnostic criteria are as follows: according to OSAHS Diagnosis and Treatment Guidelines (Revised 2011), ${ }^{[8]}$ apnea and hypopnea index (AHI) is defined as the sum of the number of sleep apnea and the number of hypopnea per hour at night; OSAHS refers to the fact that AHI $\geq 5$ times/h during the process of 7-hour sleep each night.

\subsection{Statistical methods}

SPSS 17.0 software was applied to the statistical analysis and the measurement data were represented by $\bar{x} \pm \mathrm{s}$. Analysis of variance was used in the comparison among groups and the comparison between two groups was tested by use of $q$-test. The data in OSAHS group were analyzed with the application of Pearson correlation and multivariate linear regression. The difference $(p<.05)$ was of statistical significance. 


\section{Results}

\subsection{The comparison of general information and lab- oratory indexes among 3 groups}

The levels of Hs-CRP, Hcy, CIMT, TG, TC and LDL-C in OSAHS group were higher than those in normal control group, and the level of HDL-C in the former was lower than that in the latter. The difference was of statistical signifi- cance $(p<.05)$. The levels of Hs-CRP, Hcy, CIMT, TG, TC and LDL-C in non-OSAHS group were higher than those in normal control group, and the level of HDL-C in the former was lower than that in the latter. The difference was of statistical significance $(p<.05)$. The levels of Hs-CRP, Hcy, CIMT and plaque score in OSAHS group were higher than those in non-OSAHS group, and the difference was of statistical significance (all $p<.05$, see Table 1 ).

Table 1: General information and laboratory indexes of OSAHS group, non-OSAHS group and the normal control group

\begin{tabular}{|c|c|c|c|}
\hline Indexes & OSAHS Group & Non-OSAHS Group & Normal Control Group \\
\hline $\mathrm{N}$ (male/female) & $60(35 / 25)$ & $69(38 / 31)$ & $30(16 / 14)$ \\
\hline Age (years) & $75 \pm 8$ & $73 \pm 8$ & $74 \pm 8$ \\
\hline BMI $\left(\mathrm{kg} / \mathrm{m}^{2}\right)$ & $25.4 \pm 1.7$ & $25.5 \pm 2.3$ & $25.0 \pm 1.7$ \\
\hline $\mathrm{TG}(\mathrm{mmol} / \mathrm{L})$ & $2.52 \pm 1.40^{\mathrm{a}}$ & $2.51 \pm 2.02^{1}$ & $0.99 \pm 0.24$ \\
\hline $\mathrm{TC}(\mathrm{mmol} / \mathrm{L})$ & $5.74 \pm 1.16^{\mathrm{b}}$ & $5.49 \pm 1.09^{\mathrm{m}}$ & $3.63 \pm 0.71$ \\
\hline HDL-C (mmol/L) & $1.16 \pm 0.44^{\mathrm{c}}$ & $1.25 \pm 0.41^{\mathrm{n}}$ & $1.40 \pm 0.25$ \\
\hline LDL-C (mmol/L) & $3.15 \pm 0.47^{\mathrm{d}}$ & $3.05 \pm 0.70^{\circ}$ & $2.47 \pm 0.27$ \\
\hline Hs-CRP (mg/L) & $3.40 \pm 0.91^{\mathrm{ef}}$ & $2.57 \pm 1.01^{\mathrm{p}}$ & $2.06 \pm 1.22$ \\
\hline Hcy $(\mu \mathrm{mol} / \mathrm{L})$ & $16.50 \pm 2.89^{\mathrm{gh}}$ & $11.20 \pm 2.32^{\mathrm{q}}$ & $6.04 \pm 1.85$ \\
\hline CIMT (mm) & $1.06 \pm 0.14^{\mathrm{ij}}$ & $0.93 \pm 0.13^{\mathrm{r}}$ & $0.65 \pm 0.07$ \\
\hline Plaque Score (Scores) & $1.92 \pm 1.03^{\mathrm{k}}$ & $1.35 \pm 0.97$ & \\
\hline
\end{tabular}

Note. OSAHS: obstructive sleep apnea hypopnea syndrome; Hs-CRP: hypersensitive C-reactive protein; Hcy: homocysteine; CIMT: carotid intima-media thickness. In comparison with normal control group, ${ }^{a} \mathrm{t}=5.91,{ }^{\mathrm{b}} \mathrm{t}=9.17,{ }^{\mathrm{c}} \mathrm{t}=-2.83,{ }^{\mathrm{d}} \mathrm{t}=7.47,{ }^{\mathrm{c}} \mathrm{t}=5.88,{ }^{\mathrm{g}} \mathrm{t}=18.15,{ }^{\mathrm{i}} \mathrm{t}=15.62,{ }^{\mathrm{l}} \mathrm{t}=4.09,{ }^{\mathrm{m}} \mathrm{t}=$ $8.56,{ }^{\mathrm{n}} \mathrm{t}=-1.82,{ }^{\mathrm{o}} \mathrm{t}=4.45,{ }^{\mathrm{p}} \mathrm{t}=2.20,{ }^{\mathrm{q}} \mathrm{t}=10.73,{ }^{\mathrm{r}} \mathrm{t}=11.51$, all $p<.05$; in comparison with non-OSAHS group, ${ }^{\mathrm{f}} \mathrm{t}=4.84,{ }^{\mathrm{h}} \mathrm{t}=11.72,{ }^{\mathrm{j}} \mathrm{t}=5.54,{ }^{\mathrm{k}} \mathrm{t}=3.91$, all $p<.05$

\subsection{Related factors of CIMT in OSAHS group -0.17).}

In OSAHS group, the results of correlation analysis showed that CIMT and plaque score were positively correlated to age, BMI, TG, TC, LDL-C, Hs-CRP and Hcy $(r$ values of CIMT to each index were $0.28,0.21,0.39,0.59,0.52,0.66$ and $0.76 ; r$ values of plaque score to each index were 0.18 , $0.30,0.29,0.52,0.53,0.64$ and 0.67); CIMT and plaque score were uncorrelated to HDL-C ( $r$ value of CIMT and HDL-C was $-0.15, r$ value of plaque score and HDL-C was

\subsection{Multiple-factor analysis in OSAHS group}

Taking CIMT and plaque score as dependent variables, and age, BMI, TG, TC, HDL-C, LDL-C, Hs-CRP and Hcy as independent variables, the analysis showed that age, BMI, LDL-C, Hs-CRP and Hcy were all influencing factors of CIMT and carotid plaques (all $p<.05$, see Tables 2-3).

Table 2: Multivariate linear regression analysis of CIMT-related risk factors in 60 patients of OSAHS group

\begin{tabular}{|c|c|c|c|c|c|}
\hline DV & $\bar{\beta}$ & SE & Standard Coefficient & $t$ & $p$ \\
\hline Age & 0.006 & 0.001 & 0.379 & 4.722 & .000 \\
\hline BMI & 0.015 & 0.007 & 0.189 & 2.249 & .029 \\
\hline LDL-C & 0.055 & 0.025 & 0.187 & 2.158 & .035 \\
\hline Hs-CRP & 0.039 & 0.013 & 0.260 & 2.965 & .004 \\
\hline Hcy & 0.014 & 0.004 & 0.300 & 3.304 & .002 \\
\hline
\end{tabular}

Note. OSAHS: obstructive sleep apnea hypopnea syndrome; CIMT: carotid intima-media thickness; Hs-CRP: hypersensitive C-reactive protein; Hcy: homocysteine 
Table 3: Multivariate linear regression analysis of carotid plaque related risk factors in 60 patients of OSAHS group

\begin{tabular}{llllll}
\hline DV & $\boldsymbol{\beta}$ & SE & Standard Coefficient & $\boldsymbol{t}$ & $\boldsymbol{p}$ \\
\hline Age & 0.027 & 0.012 & 0.211 & 2.185 & .033 \\
BMI & 0.150 & 0.061 & 0.249 & 2.459 & .017 \\
LDL-C & 0.513 & 0.229 & 0.234 & 2.241 & .029 \\
Hs-CRP & 0.243 & 0.119 & 0.216 & 2.040 & .046 \\
Hcy & 0.088 & 0.039 & 0.247 & 2.262 & .028 \\
\hline
\end{tabular}

Note. OSAHS: obstructive sleep apnea hypopnea syndrome; Hs-CRP: hypersensitive C-reactive protein; Hcy: homocysteine

\section{Discussion}

Patients with OSAHS nightly suffer from repeated intermittent hypoxia, leading to hypoxemia and hypercapnia, causing a series of multisystemic pathophysiologic changes, which play an active role in the progress of AS. In this study, the levels of Hs-CRP and Hcy in OSAHS group were higher than those in non-OSAHS group and normal control group; the increase of Hcy was synchronous with that of Hs-CRP, suggesting that Hcy was significantly identical to Hs-CRP. CIMT in OSAHS group was higher than that in non-OSAHS group, suggesting that OSAHS should be a factor that could aggravate AS. Correlation analysis showed that, in OSAHS group, CIMT and plaque score were correlated to age, BMI, TG, TC, LDL-C, Hs-CRP and Hcy. Multivariate linear regression analysis showed that age, BMI, LDL-C, Hs-CRP and Hcy were influencing factors of CIMT and plaque score, indicating that these factors were also influencing factors of AS. Early diagnosis and treatment for senile OSAHS patients, can delay or improve the progress of AS.

One of mechanisms concerning AS is an inflammation theory that a variety of inflammatory cytokines are involved in the mechanism of AS. This study confirms that OSAHS can promote the increase of inflammatory cytokines. Correlation analysis of OSAHS group showed that, CIMT and carotid plaques were positively correlated to TG, TC and LDL-C; multiple linear regression analysis showed that LDL-C was an influencing factor of CIMT and carotid plaques.

OSAHS is closely related to cardiovascular disease, and there are many possible mechanisms currently, but most of scholars tend to support the inflammation theory in recent years. Senile OSAHS and AS share common pathophysiological bases, one of which is vascular endothelial dysfunction, caused by inflammatory cytokines, is an important target. ${ }^{[3,9]}$ Domestic literatures have reported that Hs-CRP and Hcy are risk factors for type 2 diabetes combined with coronary disease, Hs-CRP and Hcy have an important clinical guidance significance in the early predication of early complications caused by type 2 diabetes. ${ }^{[10]}$

OSAHS, obesity, dyslipidemia, Hs-CRP and Hcy are in- fluencing factors of AS. Therefore, in the clinical practice, we can delay the progress of AS by improving hypoxemia, weight management and lipid-lowering therapy in senile OSAHS patients. Reducing the levels of Hs-CRP and Hcy to prevent or delay of AS, is also an important means of AS treatment in the future.

In the study of OSAHS and metabolic syndrome, Basoglu et al. ${ }^{[11]}$ have found that the level of Hs-CRP in OSAHS group was higher than that in control group, and OSAHS may have a causal relationship with metabolic syndrome. This study showed that OSAHS could promote the increase of Hs-CRP level in patients with cardiovascular diseases such as hypertension and type 2 diabetes, with the severity of AS increased.

Hcy is a sulphydryl-containing nonessential amino acid that does not participate in the biosynthetic process of proteins in the body. It is metabolized in cells by three pathways: remethylation, transsulfuration and direct release into extracellular fluids. It is intended to ensure the metabolic balance of sulfur-containing amino acids in the body. Both OSAHS and Hcy are considered as independent risk factors for cardiovascular disease. ${ }^{[11]}$ Thakre et al. ${ }^{[12]}$ have found that the level of Hcy in OSAHS people was significantly higher than that in non-OSAHS people, Sales et al. ${ }^{[13]}$ also came to similar results in their research. Animal models have confirmed that the level of Hcy in OSAHS rats is higher than that in non-OSAHS rats; hypoxic changes in OSAHS rats may result from the fact that increased level of Hcy enhances inflammatory responses in the body and leads to the occurrence of AS in cerebral arteries. ${ }^{[14]}$ Cintra et al. ${ }^{[15]}$ have found that, in their research, there is no difference in the level of Hcy between OSAHS and non-OSAHS groups; there is no difference in the level of Hcy between obese patients with and without OSAHS. ${ }^{[11]}$ Hence, the change in Hcy level remains to be controversial and worthy of being studied further.

\section{Conflicts of Interest Disclosure}

The authors have no conflicts of interest related to this article. 


\section{References}

[1] Janssens JP, Pautex S, Hilleret H, et al. Sleep disordered breathing in the elderly. Aging Clin Exp Res. 2000; 12(6): 417-429. https://doi.org/10.1007/BF03339872

[2] Oikawa M, Ota H, Takaya N, et al. Carotid magnetic resonance imaging A window to study atherosclerosis and identify highrisk plaques. Circ J. 2009; 73(10): 1765-1773. PMid: 19755748. https://doi.org/10.1253/circj.CJ-09-0617

[3] Lui MM, Lam DC, Ip MS. Significance of endothelial dysfunction in sleep-related breathing disorder. Respirology. 2013; 18(1): 39-46. PMid: 22712467. https://doi.org/10.1111/j.1440-1843. $2012.02212 . \mathrm{x}$

[4] Libby P. Inflammation in atherosclerosis. Arterioscler Thromb Vasc Biol. 2012; 32(9): 2045-2051. PMid: 22895665. https://doi. org/10.1161/ATVBAHA .108.179705

[5] Kuniyoshi FH, Pusalavidyasagar S, Singh P, et al. Cardiovascular consequences of obstructive sleep apnoea. Indian J Med Res. 2010; 131(2): 196-205. PMid: 20308745.

[6] Klinghammer L, Urschel K, Cicha I, et al. Impact of telmisartan on the inflammatory state inpatients with coronary atherosclerosisinfluence on IP-10, TNF- $\alpha$ and MCP-1. Cytokine. 2013; 62(2): 290296. PMid: 23541900. https://doi.org/10.1016/j.cyto. 2013.02.001

[7] Zureik M, Bureau JM, Temmar M, et al. Echogenic carotid carotid plaques are associated with aortic arterial stiffness in subjects with subclinical carotid atherosclerosis. Hypertension. 2003; 41(3): 519527. PMid: 12623953. https://doi.org/10.1161/01.HYP. 0000054978.86286 .92
[8] RDDS Group of Chinese Thoracic Society. OSAHS Diagnosis and Treatment Guidelines (Revised 2011). Chinese Journal of Tuberculosis and Respiratory Diseases. 2012; 35(1): 9-12.

[9] Han RM, Li NF, Yan ZT, et al. Analysis of influencing factors on circadian blood pressure of hypertensive patients with obstructive sleep apnea-hypopnea syndrome. Chinese Journal of Cardiology. 2013; 41(9): 751-755. PMid: 24331803.

[10] Zhang LZ, Zhou Y, Wang RX, et al. Risk factors for coronary heart disease in patients with type 2 diabetes mellitus. Chinese Journal of Geriatric Heart Brain and Vessel Diseases. 2013; 15(4): 373-375.

[11] Basoglu OK, Sarac F, Sarac S, et al. Metabolic syndrome, insulin resistance, fibrinogen, homocysteine, leptin, and C-reactive protein in obese patients with obstructive sleep apnea syndrome. Ann Thorac Med. 2011; 6(3): 120-125. PMid: 21760842. https://doi.org/ $10.4103 / 1817-1737.82440$

[12] Thakre TP, Mamtani M, Ujaoney S, et al. Association of plasma homocysteine with self-reported sleep apnea is confounded by age: results from the national health and nutrition examination survey 2005-2006. Sleep Disord. 2012; 2012: 634920. PMid: 23471122. https://doi.org/10.1155/2012/634920

[13] Sales LV, Bruin VM, D'Almeida V, et al. Cognition and biomarkers of oxidative stress in obstructive sleep apnea. Clinics (Sao Paulo). 2013; 68(4): 449-455. https://doi.org/10.6061/clinics/ 2013(04) 03

[14] Song KY, Xu P, Shi YH. Injury of cortex and hippocampus in rats with obstructive sleep apnea syndrome and its mechanisms. Chinese Journal of Geriatric Heart Brain and Vessel Diseases. 2013; 15(2): 193-196.

[15] Cintra F, Tufik S, D'Almeida V, et al. Cysteine: a potential biomarker for obstructive sleep apnea. Chest. 2011; 139(2): 246-252. PMid: 20651023. https://doi.org/10.1378/chest. 10-0667 